\title{
Variables familiares asociadas a comportamientos suicidas: revisión preeliminar de literatura
}

\section{Family variables associated with suicidal behaviors: a review of literature}

Morelia Erandeni Gil Díaz

Judith López Peñaloza

Universidad Michoacana de San Nicolás Hidalgo

Alicia Edith Hermosillo de la Torre

Universidad Autónoma de Aguascalientes

\author{
Luis Miguel Sánchez-Loyo \\ Universidad de Guadalajara
}

\section{Resumen}

El suicidio es considerado por la Organización Mundial de la Salud (2014) como un fenómeno complejo y de tipo multicausal. Esta problemática no puede ser explicada por solo una variable, dado que su presencia o desarrollo en un individuo puede deberse a múltiples factores de origen diverso. De acuerdo con los datos reportados por la Organización Panamericana de la Salud (2016), la población joven y los adolescentes son los grupos que se encuentran en mayor riesgo ante dicha problemática. El presente estudio es una revisión de literatura exploratoria sobre variables relacionadas con el sistema familiar y la presencia de los comportamientos suicidas. El objetivo principal de la presente revisión de literatura fue identificar exclusivamente aquellos artículos de investigación que se centraran en las variables del sistema familiar y la presencia de comportamientos suicidas. La búsqueda se realizó durante los meses de enero a mayo del 2019, para lo cual se consultaron cinco bases de datos. En un inicio se obtuvieron un total 60 .

Palabras clave: comportamiento suicida, ideación suicida, intento suicida, familia, revisión de literatura exploratoria.

\footnotetext{
Nota del autor

Morelia Erandeni Gil Díaz, Facultad de Psicología, Universidad Michoacana de San Nicolás de Hidalgo. Judith López Peñaloza, Facultad de Psicología, Universidad Michoacana de San Nicolás de Hidalgo. Alicia Edith Hermosillo de la Torre, Facultad de Psicología, Universidad Autónoma de Aguascalientes. Luis Miguel Sánchez-Loyo, Facultad de Psicología, Universidad de Guadalajara

La correspondencia en relación con este artículo debe dirigirse a Morelia Erandeni Gil Díaz, Elodia Romo, \#179, Col. Jacarandas, Morelia, Michoacán, México.

Correo electrónico: moregil@hotmail.com
} 


\begin{abstract}
The World Health Organization (2014) considers suicide a complex and multicausal phenomenon. As such, this phenomenon cannot be explained by only one variable and its presence or development in an individual is likely due to multiple factors of diverse origins. According to data reported by the Panamerican Health Organization (2016), children and adolescents are the groups most at risk for suicidal attempts. The present study is a review of exploratory literature on variables related to the family system and the presence of suicidal behaviors. Therefore, the main objective of this literature review was to identify and explore research articles that focused on variables related to the family and suicidal behaviors. The search was carried out during the months of January to May 2019, for which five databases were consulted, considering only research articles that focused on variables regarding family systems and suicidal behaviors. Initially, a total of 60 articles were obtained. According to the inclusion criteria for the literature review, 20 research articles were included in the present inquiry. Through a systematic review of the exploratory literature, several variables regarding family systems and suicidal behaviors were identified, such as family functioning, communication, support received from its members, and others. The absence of these variables is negatively correlated with the presence of suicidal behaviors. Familial variables, specifically those related to interactions between family members, play an important role in the prevention of suicidal behavior.
\end{abstract}

Keywords: suicidal behavior, suicidal ideation, suicidal attempt, family, exploratory literature review.

\section{Introducción}

El comportamiento suicida está considerado por diversos organismos internacionales como la Organización Mundial de la Salud (2014) y la Organización Panamericana de la Salud (2016), como una problemática de salud pública. De acuerdo con esta última, dicha problemática afecta especialmente a la población joven que se encuentra en el rango de edad de 15 a 24 años. Como señala este organismo, un número aproximado de 65000 personas fallece cada año por tal condición, únicamente en la región de las Américas. Así, los datos estadísticos de la OMS (2014) y la OPS (2016) señalan que el suicidio es la segunda causa de muerte en población joven.
De acuerdo con Borges, Orozco, Benjet y Medina-Mora (2010) en México el suicidio es considerado como una problemática de salud pública y de salud mental. Estos autores señalan que en el periodo de 1970 al 2007, el número de muertes por esta causa registró un aumento de un $275 \%$. Según estos datos epidemiológicos, los fallecimientos por estas causas se incrementan especialmente en la población joven, la cual va de 12 a 29 años.

Kleiman y Beaver, 2013; Wyatt, Ung, Park, Kwon y Shervrin, 2015; Whitaker, Shapiro y Shiels, 2016). De este modo, la problemática suicida ha sido estudiada desde diversos ángulos; como se mencionó anteriormente, esto ha permitido obtener un panorama amplio de las 
diversas variables y características del suicidio, las cuales llegan a influir en la aparición de comportamientos tales como ideación e intentos suicidas.

Este tipo de estudios resaltan que el suicidio es un problema multifactorial y complejo, el cual se origina por diversos factores que interactúan entre sí. El objetivo del presente trabajo fue realizar una revisión preliminar de literatura para identificar las diversas variables relacionadas con el sistema familiar y la presencia de comportamientos suicidas. Algunas de las variables mencionadas como factores de riesgo son problemas con el manejo de emociones, baja autoestima, consumo de sustancias, desesperanza, no contar con relaciones interpersonales o sentirse aislados, incapacidad para manejar situaciones estresantes, entre otros (Kleiman y Beaver, 2013; Wyatt, Ung, Park, Kwon y Shervrin, 2015; Whitaker, Shapiro y Shiels, 2016).

Los estudios realizados en México sobre los factores de riesgo y protección, han obtenido resultados similares a los datos internacionales: coinciden en la importancia de la presencia de factores como el adecuado manejo de las emociones o la autoregulación, la autoestima, la impulsividad, el consumo de sustancias y la presencia de sintomatología depresiva. De forma específica, en estudios con población adolescente escolarizada, se ha reportado la dificultad de manejar las emociones tales como la tristeza o el enojo, la presencia de problemas familiares, la ausencia de redes de apoyo significativas, el sentirse incomprendidos por su familia, un sentimiento de desesperanza, poca confianza con los padres y pobre comunicación con el sistema familiar además de una interacción conflictiva con el padre o la madre (Gónzalez-Forteza, Villatoro-Velázquez, Alcántar-Escalera, Medina-Mora, Fleiz, Bermudez y Amador, 2002; Gózalez, Forteza, Ramos-Lira, Caballero-Gutierrez y WagnerEcheguarray, 2003; Pérez-Amezcua, Atienzo, De Castro, Leyva-López y Chávez-Ayala, 2010; Arenas-Landgrave, Lucio-Gómez Maquoe y Forns 2012; Chávez-Hernández, GónzalezForteza, Júarez-Loya, Vazquez-Vega y JiménezTapia, 2015).

De igual forma, un estudio realizado por Rivera-Heredia y Ándrade-Pálos, (2006) encontró diferencias al realizar comparaciones en poblaciones de adolescentes con intento suicida y sin intento suicida. Halló que aquellos adolescentes que contaban con intentos suicidas presentaban disfunciones familiares, percepción de poco apoyo familiar y dificultades en los patrones de interacción entre los miembros de la familia. Las autoras identificaron problemas con el manejo del enojo y la tristeza; dificultad para recuperar el equilibrio después de enfrentar situaciones estresantes, así como también una dificultad para pedir ayuda.

\section{Método}

Se realizó una revisión preliminar de literatura bajo los criterios PRISMA (Liberati, Altman,Ttzlaff, Mulrow, Gøtzsche, Ioannidis, 
Clark, Deveraux, Kleijen, y Moher, 2009; Moher, Liberati, Tetzlaff, y Altman, 2009), específicamente para las revisiones de literatura preliminares o scoping review (Tricco, Lillie, Zarin, O〉Brien, Colquhoun, 2018).

La búsqueda para seleccionar los artículos se realizó durante los meses de enero a mayo del 2019. Los artículos se buscaron en bases de datos, tales como, Wiley, ScienceDriect, Emerald, PubMed y Scielo.

La atención se centró en aquellos artículos publicados en los años del 2010 al 2018. Como criterios de inclusión, se definió que solo serían elegibles artículos de investigación dentro de los cuales se consideraran variables de tipo familiar y la presencia de comportamientos suicidas. Como criterios de exclusión, no se consideraron para el presente trabajo artículos de metaanálisis, revisiones teóricas o sistemáticas, artículos sobre factores de riesgo o protección de la conducta suicida, artículos relacionados con la prevención del suicidio, investigaciones sobre programas de intervención ni estudios sobre posvención del suicidio. Tampoco se admitieron investigaciones que no especificaran de forma clara que los principales objetivos del estudio era el explorar o identificar las variables cuya presencia o ausencia dentro de las familias puede asociarse al desarrollo de conductas suicidas en los integrantes de esta, inluso aunque en ellos se mencionara a la familia. Se excluyeron también artículos que incluyeran como factor de riesgo antecedentes de suicidio en la historia familiar.
La búsqueda estaba delimitada por aquellos artículos científicos que se centraban específicamente en el estudio de las características de los sistemas o ambientes familiares y en la presencia o ausencia de elementos que, dentro del sistema, funcionarían como factores de riesgo asociados a la presencia o ausencia del desarrollo de conductas suicidas. De esta manera, el procedimiento para la búsqueda de los artículos se realizó tanto en inglés como en español, utilizando las palabras clave "suicidal behaviour”, "suicide ideation", "suicide attempt" "family", "family functioning" y "family support”. En la búsqueda inicial se encontraron 60 artículos; al hacer una revisión de estos (año, título y resumen), la lista se redujo a 30 artículos; después de hacer la evaluación pertinente, se incluyó únicamente 20 artículos, los cuales cumplían con los criterios de inclusión.

\section{Resultados}

El objetivo de la presente revisión preliminar de literatura era el conocer de forma inicial o exploratoria las variables familiares que se encuentran presentes para el desarrollo del comportamiento suicida, identificadas de forma sistemática y provenientes de artículos de investigación. Se encontró una diversidad de variables relacionadas específicamente con la interacción o pautas transaccionales de los integrantes del ambiente familiar. Es importante señalar que en las investigaciones revisadas eran adolescentes quienes presentaban conductas 
suicidas; las variables medidas incluían, por ejemplo la comunicación, cuestiones de apego, el funcionamiento, las interacciones entre los integrantes, el ambiente percibido, la existencia de conflictos, todas ellas acotadas al ámbito familiar. Todos los estudios reportaron una o varias de estas variables en poblaciones compuestas en su mayoría por adolescentes que, además, contaban con un historial o presencia de ideación o tentativas suicidas.

Con base en lo anterior, los resultados se pueden agrupar en diversas variables. Todas ellas están relacionadas con componentes de los sistemas familiares, que a su vez están ligados a las formas en las que los integrantes de las familias se relacionan unos con otros. Además, las variables también están vinculadas con la ausencia de elementos o características del sistema familiar, pues estos juegan un rol importante como factores de riesgo, ya que sino están presentes generan sistemas familiares que no proveen confianza, seguridad ni cuidado a sus integrantes, especialmente a los hijos. Es decir, que estos ambientes cuentan con características que los hacen parecer sistemas en los cuales el apoyo es nulo y donde pareciera que la probabilidad de encontrar ayuda es muy poca.

Los resultados de los estudios revisados se pueden agrupar en tres componentes de las familias, los cuales se desglosan a continuación:

1) Interacciones familiares: este componente del ambiente familiar fue reportado en los estudios contemplados dentro de la presente revisión de literatura; se reportó la presencia o percepción de interacciones familiares especialmente de tipo negativas señaladas. Asimismo, se encontró de forma frecuente no solo interacciones conflictivas entre padres e hijos, sino también la percepción de los hijos de interacciones conflictivas, deficientes y/o la falta de unión entre los padres. Estos estudios resaltaban la relación entre las interacciones problemáticas, deficientes y disfuncionales y la presencia de conductas suicidas y sintomatología depresiva.

Otra variable mencionada dentro de este campo fue el estilo parental (asociado con regaños o castigos severos), ligado a la percepción de una interacción poco significativa con los padres, o un desempeño poco funcional de estos percibido por parte de los hijos, quienes contaban con el historial de comportamiento suicida. De esta forma, dentro de las interacción de los integrantes del sistema familiar, se hace referencia a la importancia de la calidad de la misma percibida por los integrantes, la cual lleva a ver el sistema como pasivo, en el cual los hijos se encuentran insatisfechos. Estos convierten a este componente familiar en un factor de riesgo para el desarrollo de comportamientos suicidas, pues tal como se observó en la literatura revisada, estas conductas están presente en los hijos de este tipo de ambientes (Consolí, Peyre, Speranza, Hassler, Falissard, Touchettte, Cohen, Moro, y Revhá-Lévy, 2013; Zhai, Bai, Chen, Han, Wong, Qiao, Qiu, Young y Young, 2015).

2) Funcionamiento familiar y apego: en la literatura revisada, principalmente se menciona 
el papel que tiene este elemento al hablar de los comportamientos suicidas. Uno de los principales resultados fue que en las familias cuyos hijos percibían un mejor funcionamiento familiar se registraba también una disminución en la ideación suicida. En cuanto al componente del apego y la cohesión, se encontró que, al igual que los componentes anteriormente mencionados, pueden llegar a brindar una comprensión sobre la presencia de la conducta suicida. De acuerdo con Law y Shek, (2016) al realizar un estudio longitudinal con adolescentes se observó que, en cuanto al funcionamiento familiar, aquellos que reportaban mayores conflictos en la familia tenían mayor riesgo de presentar comportamientos suicidas $y$ autolesiones. Entonces, el contar con un funcionamiento familiar deficiente o débil, aunado a una estructura familiar inadecuada, coloca a los hijos en un mayor riesgo de presentar conductas suicidas (Zaborskis, Sirvyte y Zemaitiene, 2016).

En contraste con lo anterior, Bravo-Andrade, López-Peñaloza, De la Roca-Chiapas y Rodriguez-Morril (2016), al trabajar con una muestra de adolescentes, encontraron que el riesgo de suicidio es bajo cuando no se perciben dificultades o falta de unión en la familia, y al considerar el contexto familiar como apropiado para la expresión de emociones.

Así, en artículos se menciona que el apego juega un rol fundamental en la ideación y el intento suicida; de este modo, se encontraron resultados que reportaban que los adolescentes que presentaban intento suicida tenían puntajes bajos en relación con el apego a los padres. Con base en lo anterior, considerarse esta última como una variable predictora para los intentos suicidas en los adolescentes (Sheftall, Mathias, Furv y Dougherty, 2013).

Con respecto al funcionamiento familiar, específicamente a la cohesión y la adaptabilidad, se resalta que, al igual que con las otras variables familiares, la percepción que tiene el adolescente es la que propicia o determina la aparición de intentos suicidas. Así, al comparar adolescentes con $\mathrm{y} \sin$ intentos suicidas, se reporta que estos últimos presentan un apego menor con los padres, al igual que una baja cohesión y adaptabilidad familiar. (Pineda y Dadds, 2013; Sheftall, Mathias, Furv y Dougherty, 2013).

Retomando el concepto de apego y su función dentro de la familia como variable familiar con relación a la prevención de conductas suicidas, Maimon, Browning y Brooks-Gum (2010) señalan que el apego a la familia reduce la probabilidad de que los adolescentes presenten intentos de suicidio. Desde esta perspectiva, el apego familiar funciona como moderador de carácter protector; al estar presente, brinda apoyo, seguridad y confianza a los adolescentes. Los que cuentan con un mayor apego a los padres muestran menores puntajes de intentos suicidas, por lo que, estos autores consideran que el apego familiar es un factor protector que al estar presente regula o modera la incidencia o el desarrollo de intentos suicidas en los hijos. 
Dentro del tema de la cohesión, en un estudio realizado por Pereira, Abreu y Martins (2014) con 534 adolescentes sobre variables relacionadas con el funcionamiento familiar - como lo son la cohesión y la flexibilidad, factores protectores al hablar de riesgo suicida, específicamente de ideación suicida- se reportó que aquellos adolescentes que contaban con familias más balanceadas, es decir, con alta cohesión y flexibilidad, mostraban puntajes más bajos de ideación suicida. En este mismo sentido, Ahookhosh, Bahmaní, Agari y Moghaddom (2015) indicaron que la cohesión y la expresividad familiar actuaron como factores protectores ante la depresión, la desesperanza, la ansiedad y la ideación suicida.

3) Apoyo familiar: de acuerdo con la revisión realizada, la presencia o ausencia de esta característica en particular se relaciona de manera negativa con la presencia de sintomatología depresiva, desesperanza e ideación suicida de forma precisa en los hijos, lo cual ilustra el impacto que el ambiente familiar tiene (Chang, Chang, Martos, Sallay, Lee, Stom, Batterbee y Yu, 2017). Por esto, el componente de apoyo familiar, al estar presente en la familia de forma activa, es un factor psicológico positivo que deberá ser retomado y considerado no solo como elemento de prevención, sino también para entender los comportamientos suicidas.

Con base en lo anterior, uno de los estudios revisados resalta la importancia de trabajar con los integrantes de los sistemas familiares, con el objetivo de desarrollar o adoptar y mantener un sistema de apoyo positivo el cual funcione como recurso para la prevención de dicho fenómeno, considerando que la familia puede funcionar como una red de apoyo y ayuda de primera línea en tales problemáticas e incluso juega un rol fundamental para guiar a los hijos en especial cuando son adolescentes en búsqueda de ayuda profesional como sistema, cuando el riesgo suicida es mayor, así los padres deben estar capacitados en los signos y señales de alarma como también estar monitoreando a sus hijos a partir de tener interacciones positivas y de pasar tiempo en familia. Que les permita tener un ambiente de confianza en el cual los hijos puedan expresarse, además, de conocer la conducta y las emociones de sus hijos, los cuales les brinden pistas ante cualquier problema o alteración en las mismas (Chang, Chang, Martos, Sallay, Lee, Stom, Batterbee y Yu, 2017).

De acuerdo con lo anterior, se resalta la importancia de considerar los componentes familiares mencionados como factores de riesgo claves en la prevención del suicidio, Los resultados encontrados en los artículos revisados permiten clarificar los elementos más específicos sobre los cuales las acciones preventivas deberán incidir con el objetivo de proporcionar mejores estrategias para la atención de casos relacionados con conductas suicidas. En este mismo tema, Behzad (2016) señala la importancia de considerar a la familia para las estrategias preventivas, recordando el rol crítico que tiene como factor de riesgo ante la ausencia de las variables mencionadas. 


\section{Discusión}

El objetivo de la revisión preliminar de literatura permitió explorar de forma inicial las investigaciones centradas en las familias y su importancia para la prevención de conductas suicidas. Aunque existen diversas investigaciones sobre la temática del suicidio, la mayoría de ellas están orientadas de forma específica a los factores de riesgo para dicho fenómeno. De manera que, de manera primordial, algunas de estas se dirigen más a definir de forma general y amplia esos factores de riesgo, mientras que otras centran su atención en variables como la impulsividad, la presencia de síntomas depresivos o ansiosos, o el consumo de sustancias. Así, en la literatura científica producto de investigaciones, la temática de la familia o, de forma más específica, la de variables relacionadas con el ambiente familiar, es abordada con menor frecuencia como un factor de riesgo principal para el desarrollo de conductas suicidas en las personas.

Es importante mencionar que el estudio de la familia es más incluido como factor de riesgo en los individuos al considerarla de manera particular cuando existen familiares que han muerto por esta causa. Es decir que existe una amplia cantidad de artículos que estudia a la familia y su rol dentro de la temática suicida principalmente cuando existe el precedente de integrantes del sistema que hayan tenido una muerte por suicidio. Al realizar la búsqueda de literatura, se encuentra un número mayormente considerable de estudios como los anteriormente mencionados $y$, en menor porcentaje, investigaciones centradas únicamente en las variables familiares.

Hay que resaltar que el presente trabajo constituye una propuesta que, como su nombre lo indica, es una revisión de literatura de tipo exploratoria o preliminar que permite tener un acercamiento inicial al tipo de información científica disponible al analizar el impacto que tiene la familia como factor dentro de las temáticas del suicidio. De esta manera, se brinda un esbozo que permite identificar algunos elementos que se encuentran estrechamente cercanos a los comportamientos suicidas que incluyen una variable como lo es el sistema familiar. De este modo, con esta exploración inicial sobre los ambientes familiares, es posible rescatar el papel que tiene la familia en la prevención de dicho fenómeno, resaltando que la familia ofrece el contexto necesario para el desarrollo de los individuos y que, tal como lo muestran los resultados de los trabajos revisados, cuando este no proporciona los elementos necesarios para sus integrantes, especialmente a los hijos, surgen diversas problemáticas, las cuales tienen un impacto no solo en el desarrollo de sus integrantes sino también en su calidad de vida y salud mental.

Así que, como se mencionó en los resultados, algunas de estas investigaciones se enfocaron en características de la familia, tales como, la interacciones familiares, el apoyo familiar, el funcionamiento familiar y el apego. Muchos de estos estudios reportaron que la ausencia de 
estas variables tenía un impacto significativo en la presencia, aumento o disminución de conductas suicidas. Estas investigaciones resaltaban la necesidad de que esas variables se desarrollen o se encuentren presentes en los sistemas familiares. Estos mismos resultados proporcionan una guía relevante para trabajos futuros que en lugar de enfocarse en temas de factores de riesgo y protección, los cuales están ampliamente abordados, se orienten hacie la prevención con una perspectiva dirigida al diseño de estrategias que intervengan de forma directa en los sistemas familiares, mejorándolos o fortaleciéndolos de forma que sirvan para el fortalecimiento y desarrollo de las habilidades necesarias para sus integrantes. (Maimon, Browning y Brooks-Gum, 2010; Piena y Dadds, 2013; Sheftall, Mathias, Furv y Dougherty, 2013; Zhai, Bai, Chen, Han, Wong, Qiao, Qiu, Young and Young, 2015; Chang, Chang, Martos, Sallay, Lee, Stom, Batterbee y Yu, 2017).

De esta forma, con base en la revisión de literatura, se puede apreciar cómo los sistemas familiares y las diversas variables que los constituyen influyen en el desarrollo de comportamientos suicidas en los hijos. Aunque la familia no es considerada como un factor directo de riesgo en el comportamiento suicida, es importante conocer aquellas variables que de acuerdo con algunos de los estudios anteriormente mencionados se han identificado como presentes en su desarrollo, especialmente al hablar de suicidio en adolescentes. No hay que olvidar que en este grupo de edad la familia juega un rol importante como un sistema que debe brindar protección, crecimiento y apoyo a sus integrantes. Es importante considerar que la familia, como un grupo social, tiene diferente impacto a nivel cultural, dado que tiene más influencia en algunas culturas que en otras.

En conclusión, la familia, además de poder ser denominada como factor de protección o riesgo, es una variable fundamental en la cual es necesario enfocarse. Se requiere trabajar para lograr espacios o contextos familiares que promuevan la salud, las interacciones sanas y de confianza entre sus integrantes, permitiéndoles, especialmente a los hijos, desarrollar una mejor capacidad para resolver problemas de la vida. Es de vital importancia construir contextos familiares con las habilidades necesarias para responder a las necesidades del adolescente, además de promover su autonomía, espacios a los cuales los miembros puedan recurrir en busca de ayuda. En conclusión, los sistemas familiares cuentan con diversas funciones, las cuales tienen un impacto fundamental al hablar del desarrollo de conductas de riesgo y la salud mental.

\section{Referencias}

Arenas-Landgrave, P., Gómez-Maqueo, E. L., \& Forns, M. (2012). Indicadores diferenciales de personalidad frente al riesgo de suicidio en adolescentes. Revista Iberoamaericna de Diagnóstico y Evaluación Psicológica, 1(33), 51-74. Recuperado de: https://www.aidep. org/sites/default/files/2018-12/r33art3_1. pdf. 
Ahookhosh P., Bahmani B., Asgari A., \& Hassanian Moghaddam, H. (2017). Family Relationships and Suicide Ideation: The Mediating Roles of Anxiety, Hopelessness, and Depression in Adolescents. International Journal High Risk Behaviours Addiction, 6(1). doi:10.5812/ijhrba.31573

Borges, G., Orozco, R., Benjet, C., \& MedinaMora, M. A. (2010). Suicidio y conductas suicidas en México: retrospectiva y situación actual. Salud pública. 52(4), 379389. Recuperado de: http://www.scielo. org.mx/scielo.php?script=sci_arttext\&pid $=$ S0036-36342010000400005

Behzad, D. (2016). Suicide Attempt: Risk Factors \& Family Function. Hormozgan Medical Journal, 20(2), 144-149. Recuperado de: https://pdfs.semanticscholar.org/a7af/ ba2c6e68c1efb85731dac8b3f4c32cfe925d. pdf

Bravo-Andrade, H., López-Peñaloza, J., De la Roca-Chiapas, J. M., \& Rodriguez Morrill, E. (2016). Relaciones intrafamiliares y riesgo suicida en adolescentes. Uaricha Revista de Psicologia. (13), 45-55. Recuperado de: https://www.researchgate. net/publication/324228018_Relaciones_ intrafamiliares_y_riesgo_suicida_en_ adolescentes/citation/download

Chang, E., Chang, O., Martos, T., Sallay, V., Lee, J., Stam, K., Batterbee, C., \& Yu, T. (2017). Family Support as a Moderator of the Relationship Between Loneliness and Suicide Risk in College Students: Having a Supportive Family Matters! The Family Journal, 25(3), 257- 263. Recuperado de: http://journals.sagepub.com/doi/abs/10.1177 /1066480717711102\#articleCitationDownlo adContainer

Chávez-Hernández, A. M., González-Forteza, C., Juárez Loya, A., Vázquez Vega, D., \& Jiménez Tapia, A. (2015). Ideación y tentativas suicidas en estudiantes del nivel medio del estado de Guanajuato, México. Acta Universitaria, 25(6), 43-50. doi:10.15174/au.2015.786

Chesin, M., Sonmez, C., Benjamin-Phillip, C., Beeler, B., Brodsky, B., \& Stanley, B. (2015). Preliminary Effectiveness of Adjunct Mindfulness-Based Cognitive Therapy to Prevent Suicidal Behavior in Outpatients Who Are at Elevated Suicide Risk. Mindfulness, (6), 1345-1355. doi:10.1007/ s12671-015-0405-8

Consolí, A., Peyre, H., Speranza, M., Hassler, C., Falissard, B., Touchette, E., Cohen, D., Moro, M., \& Révah-Lévy, A. (2013). Suicidal behaviors in depressed adolescents: role of perceived relationships in the family. Child and Adolescent Psychiatry and Mental Health, 7(8). doi: 10.1186/1753-2000-7-8

González-Forteza, C., Villatoro, J., Alcántar, I., Medina-Mora, M. E., Fleiz, C., Bermúdez, P., \& Amador, N. (2002). Prevalencia de intento suicida en estudiantes adolescentes de la Ciudad de México: 1997 y 2000. Salud Mental, 25(6), 1-12. 
González-Forteza, C., Ramos-Lira, L. Caballero, M. A., \& Wagner-Echeagarray, F. (2003). Correlatos psicosociales de depresión, ideación suicida e intento suicida en adolescentes mexicanos. Psicothema, 15(4), 524-532. Recuperado de: http://www. psicothema.com/pdf/1102.pdf

Gouveia-Pereira, M., Abreu, S., \& Martins, C. (2014). How do families of adolescents with suicidalideationbehave? Psicologia:Reflexão e Crítica, 27(1), 171-178. https://dx.doi. org/10.1590/S0102-79722014000100019

Kleiman, E., \& Beaver, J. (2013). A meaningful life is worth living: Meaning in life as a suicide resiliency factor. Psyachiatric research, (210), 934-939. Recuperado de: http:// ac.els-cdn.com/S0165178113004460/1 s2.0-S0165178113004460-main.pdf? tid=92f2c38a-4279-11e7-8264-00000aacb36 0\&acdnat $=1495847738 \_690 \mathrm{~b} 1 \mathrm{ce} 18 \mathrm{e} 1 \mathrm{afe} 05$ 72430b549f3e5ade

Liberati, A., Altman, D.,Ttzlaff, J., Mulrow, C., Gøtzsche, P., Ioannidis, J., Clarke, M., Deveraux., P.J., Kleijen, J., \& Moher, D. (2009). Preferred reporting items for systematic reviews and meta-analyses: the PRISMA statement. BMJ (309). https://doi. org/10.1136/bmj.b2700

Law, B., \& Shek, L. (2016). A6-year Longitudinal Study of Self-harm and Suicidal Behaviors among Chinese Adolescents in Hong Kong. Journal of Pediatric and Adolescent Gynecology, 29(1), 38-48. Recuperado de:
https://www.jpagonline.org/article/S10833188(15)00347-2/fulltext

Moher, D., Liberati, A., Tetzlaff, J., \& Altman, D. (2009). Preferred reporting items for systematic reviews and meta-analyses: the PRISMA statement. BMJ, (339). https://doi. org/10.1136/bmj.b2535

Maimon, D., Browning, C. R., \& BrooksGunn, J. (2010). Collective Efficacy, Family Attachment, and Urban Adolescent Suicide Attempts. Journal of Health and Social Behavior, 51(3), 307-324. doi: $10.1177 / 0022146510377878$

Oppenheimer, C. W., Stone, L. B., \& Hankin, B. L. (2018). The influence of family factors on time to suicidal ideation onsets during the adolescent developmental period. Journal of psychiatric research, 104, 72-77. doi:10.1016/j.jpsychires.2018.06.016

Pérez-Amezcua B., Rivera-Rivera L., Atienzo E.E., De Castro F., Leyva-López A., \& Chávez-Ayala R. (2010). Prevalencia y factores asociados a la ideación e intento suicida en adolescentes de educación media superior de la República mexicana. Salud Publica, 52, 324-333. Recuperado de: https://www.scielosp.org/article/ssm/ content/raw/?resource_ssm_path $=/$ media/ assets/spm/v52n4/v52n4a08.pdf

Pineda, J., \& Dadds, M. (2013). Family Intervention for Adolescents With Suicidal Behavior: A Randomized Controlled Trial and Mediation Analysis. Journal of the 
American Academy of Child and Adolescent Psychatry, 52(2), 851-862. https:/doi. org/10.1016/j.jaac.2013.05.015

Organización Panamericana de la Salud (OPS). (2016). Prevención de la conducta suicida. Recuperado de: http:// iris.paho.org/x mlui/bitstream/hand le/123456789/31167/9789275319192-spa. pdf? sequence $=1 \&$ isAllowed $=\mathrm{y}$

Organización Mundial de la Salud (OMS). (2014). Prevención del suicidio: un imperativo global. Washington, EUA: Organización Panamericana de la Salud.

Rusu, I., Cosman, D., \& Nemes B. (2012). Family-protective factor to prevent suicidal behavior in adolescents. International Journal of Bioflux Society, 4(3). Recuperado de: http://www.hvm.bioflux.com.ro/ docs/2012.103-106.pdf

Rivera-Heredia, M. E., \& Andrade-Palos, P. (2006). Recursos individuales y familiares que protegen al adolescente del intento suicida. Revista Intercontinental de Psicología y Educación, 8(2). 23-44. Recuperado de: http://www.redalyc.org/pdf/802/80280203. pdf.

Sheftall, A. H., Mathias, C. W., Furr, R. M., \& Dougherty, D. M. (2013). Adolescent Attachment Security, Family Functioning, and Suicide Attempts. Attachment and Human Development, 15(4), 368-383. doi:1 $0.1080 / 14616734.2013 .782649$

Tricco A.C., Lillie E., Zarin W., O’Brien K.K., Colquhoun H., Levac D., \& Straus, S. (2018).
PRISMA Extension for Scoping Reviews (PRISMA-ScR): Checklist and Explanation. Annual Internal Medicine, 169, 467-473. doi:10.7326/M18-0850

Withaker, K., Shapiro, V., \& Shields, J. (2016). School-Based Protective Factors Related to Suicide for Lesbian, Gay, and Bisexual Adolescents. Journal of adolescente health, (58), 63-68. Recuperado de: http:// ac.els-cdn.com/S1054139X1500364X/1s2.0-S 1054139X1500364X-main.pdf? tid=c99893f0-427a-11e7-a962-

Wyatt, L., Ung, U., Park, R., Kwon, S., \& Shevrin, C.H. (2015). Risk factors of suicide and depression among Asian American, native Hawaiian, and Pacific Islander youth: a systematic literature review. Journal of Health Care Poor Underserved, 6(20), 191237. doi:10.1353/hpu.2015.0059.00000aab0 f02\&acdnat $=1495848259 \_5 \mathrm{db} 6 \mathrm{f} 27 \mathrm{e} 0694 \mathrm{a} 4$ $3433856 b 87 d 99 f 942 a$

Zhai, H., Bai, B., Chen, L., Han, D., Wang, L., Qiao, Z., \& Qiu, X.. (2015). Correlation between Family Environment and Suicidal Ideation in University Students in China. International Journal of Environmental Research and Public Health, 12(2), 14121424. doi:org/10.3390/ijerph120201412

Zhao, S., \& Zhang, J. (2015). Suicide Risks among Adolescents and Young Adults in Rural China. International Journal of Environmental Research and Public Health, 12(1), 131-145. Recuperado de: 
https://www.ncbi.nlm.nih.gov/pmc/articles/ PMC4306853/

Zaborskis, A., Sirvyte, D., \& Zemaitiene, N. (2016). Prevalence and familial predictors of suicidal behaviour among adolescents in Lithuania: a cross-sectional survey 2014. BMC Public Health. 16(554), 1-15. doi:10.1186/s12889-016-3211-x

Recibido: 09/08/2019

Revisado: 25/11/2019

Aceptado: 02/12/2019 